\title{
Editorial
}

\section{Further Thoughts on Authorship: Gift Authorship}

\author{
Richard A. Brand MD
}

Published online: 31 July 2012

(C) The Association of Bone and Joint Surgeons( 2012

Authorship is sometimes one of the most vexing ethical issues in publishing scientific articles although not a new concern. Alexander, in 1953, noted the trend in increasing numbers of authors as a result of the evolution from individual researchers to teams [1] and the complexities of establishing who should and who should not be considered an author. (A PubMed search at the time of this writing listed 778 articles with "authorship" in the title, 253 of which had been published in the past 5 years.) Several years ago Clinical Orthopaedics and Related Research published an editorial, "Thoughts on Authorship," describing recent national and international efforts to establish standards for determining authorship [3]. Part of the reason for these standards was the growing numbers of authors on most papers and the corresponding difficulty of determining the contributions of those authors. Clearly any given study and paper involves a finite amount of effort, and the larger the number of authors, the smaller percentage effort on average will be for a given author [4, 5]. However, most studies and reports likely involve substantial efforts from a few individuals with lesser contributions from others; as authors are added, at some point the effort of the additional authors will be vanishingly

The author certifies that he has no funding or commercial associations (eg, consultancies, stock ownership, equity interest, patent/licensing arrangements, etc) that might pose a conflict of interest in connection with the submitted article.

All ICMJE Conflict of Interest Forms for authors and Clinical Orthopaedics and Related Research editors and board members are on file with the publication and can be viewed on request.

R. A. Brand ( $\square)$

Editor-in-Chief, Clinical Orthopaedics and Related Research, 1600 Spruce Street, Philadelphia, PA 19103, USA

e-mail: dick.brand@clinorthop.org small or absent. The inclusion of individuals with minimal or no input reflects "loose authorship," [2], "honorary authorship" [15], or "gift authorship" [6, 8, 11]. Marcia Angell (a former Deputy Editor of New England Journal of Medicine) in her 1983 article, "Editors and Fraud" [2], referred to "loose authorship" as a form of deception. While gift authorship is not new, it appears in the literature as a major concern only in the 1980 s, perhaps owing to the continuing rise in numbers of authors and an increased sensitivity to ethical issues in publishing.

Alexander [1] likely first addressed multiple authorship and gift authorship in a serious way:

"Of even greater importance is the degrading effect that multiple authorship has upon the meaning of authorship. It would seem safe for the reader to assign a significant degree of credit to the author's name that appears first. In some instances, the reader also may be able to identify the name of the senior investigator in the laboratory of origin, and rightly or wrongly, assign him some credit for the publication. What of the rest? Just what is the reader supposed to deduce from the fact that John Doe was the seventh out of eight authors? This deduction becomes all the more difficult if the reader discovers that the work reported could easily have been accomplished by two competent investigators in two or three months' time."

Echoing Alexander's comments many years later, Jane Smith, a Deputy Editor at BMJ suggested, "Perhaps most importantly, those who have really done the work have an interest in seeing their role is not devalued by the inclusion of many who have done so little" [11]. She also noted the case of a paper with fraudulent data in which the name of a head of a department was included, although the department head acknowledged, "I was not part of this work," 
and further commented, "The head of department's name is always put on reports out of politeness." Smith concluded, "... the events (referring to the fraudulent paper) should remind 'authors' that signing their names to something they can't defend is not in their best interest." More recently, the bioethicists Smith and Williams-Jones [10] stated, "Granting authorship to everyone who enables research would be an unrealistic and extreme extension of the notion of authorship ... such authorship practices would arguably undermine the value of being named on a scientific publication." Any author should have an adequate working knowledge of the content to publically defend the work. Stewart and Feder [15] extensively documented another case of fraud, in which there were honorary authors. They argued, “... honorary authorships falsify the assignment of responsibility for published research and increase the likelihood that inaccurate data will be published." Stephen Lock (at the time Editor of the $B M J$ ) in a followup editorial to the analysis of Stewart and Feder, referred to "disturbing lapses from accepted scientific standards" including " 'gift' authorship" [9]. Grist commented, "An author is responsible for what is written, for its truth and intelligibility" [7].

While gift authorship has been increasingly recognized by the changes in international standards, Grieger suggested the introduction of international standards, such as the International Committee of Medical Journal Editors (ICMJE) guidelines, had not appeared to have influenced authors' conduct [6]. I am unaware of any data on the subject that might document Greiger's assertion, but if correct, it is disturbing.

No one would argue that determining authorship is always straightforward, particularly with the growth of larger and larger research teams and the emergence of large collaborative study groups. Standards such as those suggested by the ICMJE [16] provide guidance on criteria for authorship, but there remains considerable ambiguity and judgment must be exercised.

Several difficult issues arise with authorship, particularly in academia, where a research team may be large and somewhat fluid. First, it is natural and expected that senior authors (typically mentors to students) would include students (including residents and fellows) in their research efforts. The best educational experiences arise when a student is involved in the entire process from the initial inception of a project though publication. Participation in only a portion of a project diminishes the educational experience considerably and at some point renders a student ineligible for authorship (ie, not meeting international and/or individual journal criteria). A common scenario is when a student, who might be available for only a short time (weeks to months), is assigned to work on a project underway or perhaps even completed and submitted or conditionally accepted for publication. The student is deprived of the opportunity to see how a project is initially conceived and modified with time. If students are not substantively involved in multiple elements, they not only miss the educational opportunity, but will not fulfill criteria for authorship. To add their names is a form of gift authorship: a reward for participation in a study whether substantive or not. Authorship should not be a reward for any form of participation, although individuals with lesser participation can and should be noted in Acknowledgments. Mentors should ensure students have the opportunity to learn from a whole experience.

A second frequent issue is the role of a large number of collaborators who contribute in one way or another to a study or group of studies, but frequently not in a substantive way to a given manuscript. Sometimes these collaborators have created formal study groups for the explicit purpose of researching a group of questions in a particular field. They may meet on a more or less regular basis with group participation in designing studies and perhaps contributing data. As noted above, any given paper reflects a finite amount of work albeit varying considerably. The percentage effort of each person will on average diminish with increasing numbers of authors, sometimes to a vanishingly small effort, even for a large study [4, 5]. For any given paper there are "prime movers," typically one to four individuals who spearhead the effort. Other individuals might be considered gift authors. One solution is "collaborative" authorship. In this model, the key one to four or five authors are listed followed by the name of the group. Individuals in the group can be acknowledged several ways, including Acknowledgments at the end of the text or a footnote on the title page. In PubMed, the name of the group can contain a link which will lead to the names of the various contributors. As an alternate solution, some journals have abandoned the concept of authorship and rather embraced the concept of "contributorship" $[12,13]$.

A third sometimes difficult issue is the role of a senior person under whose aegis the work is completed. This person might have originally suggested the general idea and provided the resources (laboratory, administrative, technicians, grant support) and perhaps nominal oversight for the project, but otherwise was minimally involved (if at all) in the specific project and ultimate manuscript. Springer, the publisher of this journal, notes in their authorship guidelines, "Acquisition of funding...or general supervision of the research group alone does not constitute authorship" [14], While such support is critical, these individuals should be noted in Acknowledgements, since they would not fulfill international criteria for authorship.

A fourth issue relates to the individual contributing patients or data to a study, or collecting data or contributing technical expertise, but with minimal or no involvement 
otherwise. Determining the need even for crucial technical data (ie, a study design) does not typically require the expertise of those who might contribute such data. Such expertise might involve performing an assay and contributing crucial data, or performing critical statistical analyses, but again, these individuals should be acknowledged rather than listed as authors if those were their sole contributions.

Finally, a research team will occasionally add an author when a paper is submitted for revision but with little or no justification. One can reasonably presume authorship was discussed at the time of initial submission of a manuscript if not at the inception of a project. Given a requirement that all authors approve a submission, it seems unlikely a group of contributors would have overlooked a colleague making crucial contributions and fulfilling criteria for authorship. Therefore addition of an author would be warranted only if a reviewer and/or editor requested substantial new data and analyses and the added individual would be involved not only in the collection of that new data, but also the interpretation of the new analyses, and subsequent changes to the text. Even in this case, presumably the original contributors would have the expertise to collect and interpret the new data, much as they did for the data originally submitted. We have experienced the situation where a corresponding author asked to make relatively straightforward revisions handed the paper to a student or fellow and asked them to make the revisions, then expected that individual would become an author. Clearly, such an individual would not fulfill criteria for authorship, and the educational experience for the student would be limited. It is the responsibility of the original contributors to revise manuscripts, not additional individuals with little or no other participation in the study.

There are no simple solutions to ascertaining authorship. Smith and Williams-Jones recently commented,

"Allocation of authorship should be fair to ensure that individuals are acknowledged appropriately for their responsibility and contribution to a publication. But the nature of large group multidisciplinary health sciences research means that there are few if any well defined, agreed upon standards to support a determination of what constitutes 'fair authorship' in multi-authored studies. This lack of consensus can, at a minimum, lead to conflict between researchers about appropriate authorship (eg, what norms are best, who should be named). But this situation may also encourage unethical publication practices, such as ghost-authorship (where the author of the paper is not named) or gift-authorship (where individuals who did not contribute are named as authors), practices that have received much attention in the public and scientific press in recent years, and which contribute to scepticism about the integrity of scientific research and publications..." [10].

One important key is to discuss authorship at the outset of a project, determine how many papers might arise, and who will contribute to each and how they will contribute. Authorship should be discussed periodically as a research project advances, and again before drafting an initial manuscript since by the very nature of research some things will change. By the time a manuscript is finalized and before submission, authorship should be clearly established for the version to be submitted and considering potential minor or major revisions.

Alexander, in 1953, concluded, “A reversal of present trends will require the stringent elimination of the practice of carelessly offering co-authorship to one's colleagues as a token for small services rendered in the conduct of research" [1]. In my earlier article, I noted, "It would be as unethical to exclude an individual who materially and substantially participated in multiple elements of a study and report as it would be to include those who did not" [3]. The important consideration for authors of multipleauthored manuscripts is to carefully consider who made material and substantial contributions to multiple elements in the study, and to use other approaches to acknowledge the contributions of those who participated in a lesser way.

Acknowledgments I thank Paul Lotke MD and Lee Beadling, who critically reviewed the drafts of this manuscript and made suggestions.

\section{References}

1. Alexander RS. Trends in authorship. Circ Res. 1953;1:281-283.

2. Angell M. Editors and Fraud. CBE Views. 1983;6:3-8.

3. Brand RA. Editorial: Thoughts on authorship. Clin Orthop Relat Res. 2008;466:1002-1005.

4. Davis PJ, Gregerman RI. Parse analysis: a new method for the evaluation of investigators' bibliographies. N Engl J Med. 1969; 281:989-990.

5. Davis PJ, Gregerman RI. Parse analysis II: a revised model that accounts for phi. N Engl J Med. 1995;332:965-966.

6. Grieger MC. Authorship: an ethical dilemma of science. Sao Paulo Med J. 2005;123:242-246.

7. Grist NR. Multiple authorship. BMJ. 1989;298:748.

8. Jackway P, Boyce R. Gift co-authorships: a tangled web. Aust Clin Rev. 1990;10:72-75.

9. Lock S. Fraud in medicine. BMJ. 1988;296:376-377.

10. Smith E, Williams-Jones B. Authorship and responsibility in health sciences research: a review of procedures for fairly allocating authorship in multi-author studies. Sci Eng Ethics. 2012; 18:199-212.

11. Smith J. Gift authorship: a poisoned chalice? Not usually, but it devalues the coinage of scientific publication. BMJ. 1994;309: 1456-1457. 
12. Smith R. Authorship is dying: long live contributorship. BMJ. 1997;315:696.

13. Smith R. Let's simply scrap authorship and move to contributorship. BMJ. 2012;344:e157.

14. Springer. Authorship guidelines. 2010. Springer. Available at: http://www.springer.com/authors?SGWID=0-111-6-888221-0. Accessed July 3, 2012.
15. Stewart WW, Feder N. The integrity of the scientific literature. Nature. 1987;325:207-214.

16. Uniform Requirements for Manuscripts Submitted to Biomedical Journals: Writing and Editing for Biomedical Publication. 2008. International Committee of Medical Journal Editors. Available at: http://www.icmje.org/. Accessed July 30, 2009. 